\title{
Micromechanical Finite Element Method Modeling of Unidirectional CFRP Surface Damage
}

\author{
Vitalii Kolesnyk $^{1}$, Dmytro Kryvoruchko ${ }^{2}$, Stanislav Shvets ${ }^{3}$, Oleksandr Bublyk ${ }^{4}$ \\ v.kolesnik@ @mvi.sumdu.edu.ua ${ }^{1}$, dmytro.kryvoruchko@gmail.com $^{2}$, \\ shvetssta@gmail.com $^{3}$, bublyk.aleksandr@gmail.com ${ }^{4}$ \\ 1,2,3,4 Sumy State University, Faculty of Technical Systems and Energy Efficient Technologies, \\ 2 Rymskogo-Korsakova St., 40007 Sumy, Ukraine;
}

\begin{abstract}
Current article was devoted to FEM modelling of damages machined surface of unidirectional CFRP (carbon fiber reinforced plastic). Modelling was performed on micromechanical level. Based on the proposed spatial arrangements of fibers relative to the cutting edge and the direction of main motion to convert 3D-process of drilling CFRP in 2D- equivalent orthogonal cutting. During numerical simulation, it was found that the angle of fibers orientation affects the average value of cutting force, amplitude of oscillation and depth of damage at the machined surface. The smallest impact on machined surface damage was detected at samples with angle of fiber orientation $\theta=45^{\circ}$, which was proved in experimental study.
\end{abstract}

Keywords: CFRP, Finite element method, Micromechanical model, Orthogonal cutting, Carbon fiber, Surface damage.

\section{Introduction}

Fiber composites have characteristic feature, namely the possibility of oriented physical properties and thus CFRPs have complex mechanical, thermal, magnetic, etc. behavior when applying the load. The specific material micro-configuration and anisotropy increases the difficulty of modeling the efficient computational model. To simulate the machining process and to predict e.g. magnitude of cutting forces, wear, temperature, etc., it is possible to use the specialized commercial FEM software (more in [1], [2]). However, in generally, the machining process is characterized by many physical changes that are difficult to describe mathematically and obtain the reliable computational results consistent with the experimental data [3]. In addition, these calculations require high computing power and expensive software with a quality computing analyst.

The composite behavior and material properties are predicted either by Unit Cell (UC) for periodic or by Representative Volume Element (RVE) for random composite structure. Geometry of RVE is estimated statistically. RVE and UC are finite domains with specific boundary conditions that can be solved as continuum mechanics problem. In case of any irregularity (curved fibers) and/or randomness, the cell (volume element) can involve thousands of fibers/nanotubes. It causes huge number of equations to be solved in case of classical numerical methods (Finite Element Method (FEM) or Boundary Element Method $(\mathrm{BEM}))$ due to fine mesh. There are authors/researchers who have developed a continuum models with micro/nanofibers/particles to simulate the machining behavior in drilling and milling of such composites (more in [4], [5]). When machining CFRP, the several machining defects as delamination, burrs and subsurface changes appears [4].

\section{Methodology of research}

It is proposed to study influence of fiber orientation on defect value at machined surface of carbon fiber reinforced plastic (CFRP) samples. Study consisted from two stages: finite element modelling (FEM) of CFRP orthogonal cutting; experimental verification of FEM results.

To simulate 3D process of CFRP drilling as 2D process of orthogonal cutting the spatial model of carbon fiber orientation to the minor cutting edge of the drill and the direction of 
primary motion was proposed. FEM of unidirectional CFRP orthogonal cutting was realized at the micromechanical level.

CFRP samples for experimental orthogonal cutting was manufactured by hand lay-up technique using the technology of vacuum forming techniques in according to GOST 25.604-82, GOST 11262-80, GOST 4651-82 methodology. CFRP samples consisted of high module carbon LU-P/ 0.1GOST 28006-88The material of matrix was epoxy resin Lorit 285. Mass fraction of carbon fiber in the CFRP sample was $60 \%$, the thickness of a single layer of fibers was $0,15 \mathrm{~mm}$ and the average diameter of the fiber $-10 \mu \mathrm{m}$.

In according to GOST 11262-80 and GOST 4651-86 methodology the physical and mechanical properties of epoxy Lorit $285: E=1.4 \mathrm{MPa} ; \mu=0,45 ; \sigma_{0}=56 \mathrm{MPa} ; \sigma_{v}=67 \mathrm{MPa}$; and CFRP: $E=422 \mathrm{MPa} ; \mu=0.3 ; \sigma_{v}=450 \mathrm{MPa}$ was defined. The average coefficient of friction of CFRP and epoxy resin were identified during tribological studies. It was found that in a couple of carbide/ CFRP- 0.21 , and carbide/epoxy resin -0.19 , respectively. Defined physical, mechanical and tribological properties of carbon fiber were considered when creating a finite-element model of orthogonal cutting of CFRP.

The aim of experimental study was to define the influence of carbon fiber orientation on the defects of machined surface of CFRP samples during orthogonal cutting.

\section{Finite element modeling of CFRP machining}

\subsection{Theoretical basic of transfer from 3D to 2D cutting of CFRP fiber}

The FEM modeling was realized at micromechanical level. To transform the 3D process of drilling CFRP in 2D process of orthogonal cutting the spatial scheme of fiber orientation relatively to minor cutting edge of the drill and main motion when cutting was designed. Correlation of three angles which characterized fiber orientation in chosen coordinate system was described in according to the equation (1) and (2):

$$
\begin{aligned}
& \bar{l}=(\cos \theta, \cos \chi, \cos \eta), \\
& \operatorname{tg} \theta_{0}=\frac{\cos \chi}{\cos \theta},
\end{aligned}
$$

where $\theta, \chi, \eta$ - Euler angles of the fibers in the static coordinate system whose $\mathrm{Z}$ axis is oriented along the main motion $\left(\mathrm{D}_{\mathrm{r}}\right)$, and the $\mathrm{X}$-axis lies in the cutting plane. The analysis of variation of angle $\Theta_{0}$ of the rotational drill showed that the smaller the angle $\varphi$ in terms of the more cutting edge range of variation of the angle, the less it depends on the output angle $\theta$ (Fig. 1). $\mathrm{P}_{\tau}$ - tool orthogonal plane, $\mathrm{P}_{\mathrm{n}}$ - cutting plane, $\mathrm{P}_{\mathrm{v}}$ - basic plane, $\mathrm{A} \gamma$ - rake face of cutting tool, $\gamma$ - rake angle.

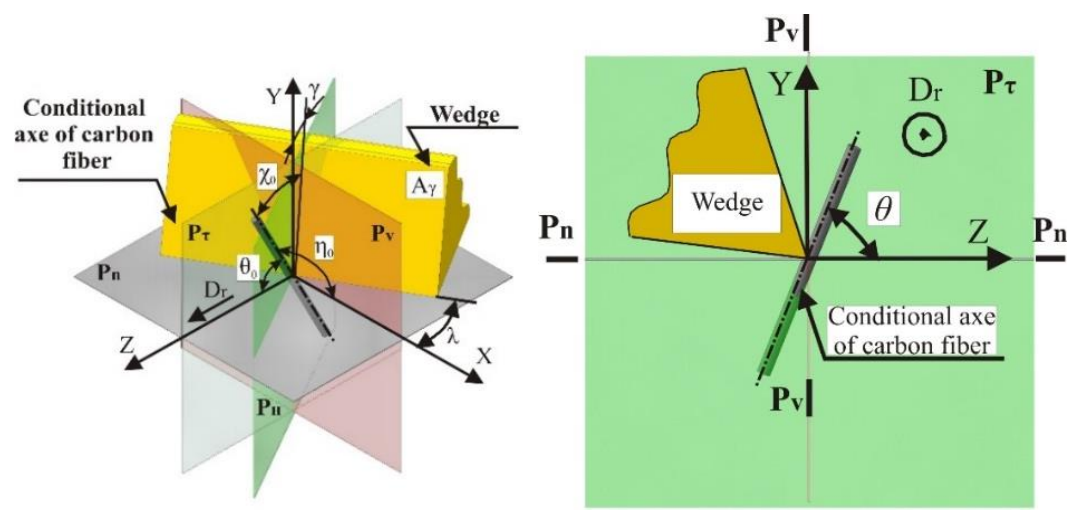

Fig. 1. Schema of fiber orientation.

So, when $\theta=0^{\circ}$, angle $\Theta_{0}$ varies from 0 to $360^{\circ}$. The angle between conditional axe of carbon fiber and minor cutting edge is $0^{\circ}, 45^{\circ}, 90^{\circ}, 135^{\circ}$ (Fig. 2). 

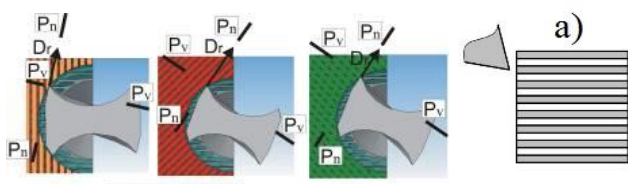

c)
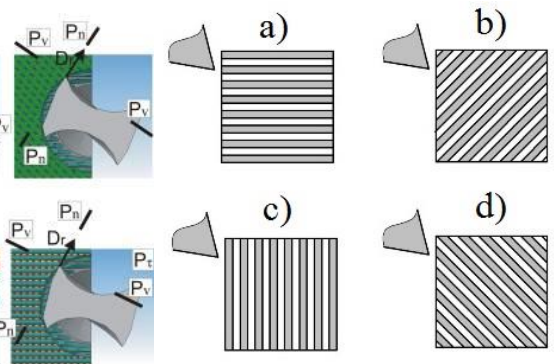

d)

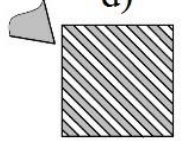

Fig. 2. The specific position of minor cutting edge to fiber

\subsection{Boundary conditions of finite element model of cutting CFRP}

Design model of CFRP for FEM modeling has following geometric characteristics width $\mathrm{b}=0.13 \mathrm{~mm}$, length $1=0.5 \mathrm{~mm}$ and a height $\mathrm{h}=0,5 \mathrm{~mm}$ (Fig. 3). Thickness of cut (a) is set equal to $0.1 \mathrm{~mm}$. Size of finite element in mesh was set $10 \mu \mathrm{m}$ in according to diameter of carbon fiber. Size of finite element for wedge was set equal to $50 \mu \mathrm{m}$.

Design model was created with three-dimensional finite elements of the first order with a uniform distribution of stresses in the volume of the element. The criterion fiber failure was value of maximum main stresses, for matrix the criterion of failure was value of accumulated plastic strain. The interaction between CFRP fiber and epoxy matrix was set completely rigid. Wedge was set completely solid. Model of fiber failure - ELASTIC; Model matrix failure PIECEWISE_LINEAR_PLASTICITY. The model of contact interaction between wedge and CFRP was ERODING_SURFASE_TO_SURFASE. Quantity of finite elements of design model was - 32532 pieces. Quantity of finite elements of workpiece - 32500 pieces. Quantity of finite elements of the tool -32 pieces.

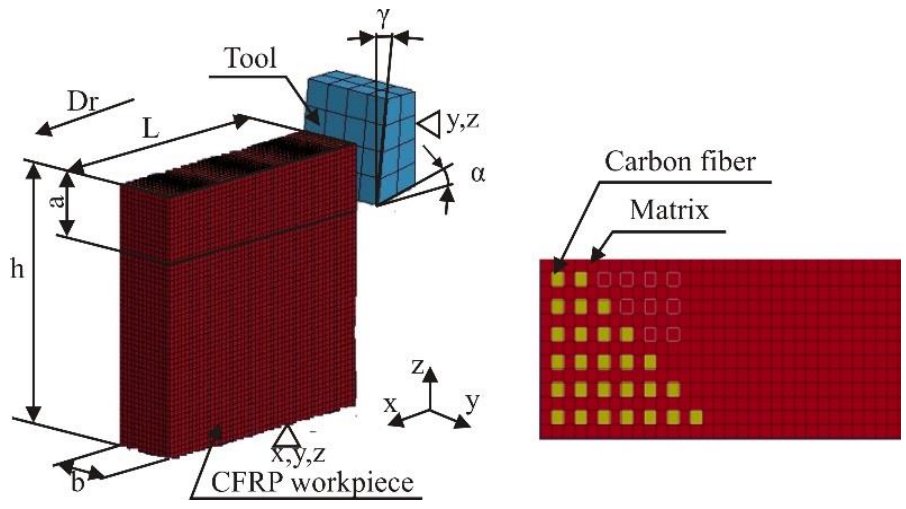

Fig. 3. Schematic of boundary conditions of FEM

As a result of the FEM modelling revealed four main stages of machining of CFRP. At first stage cutting edge just touches the surface. At the second step the cutting edge starts cutting layer of CFRP, followed by increase in cutting force. The third stage is characterized by steady-state work tool and fluctuating cutting forces ranging from 80 to $130 \mathrm{~N} / \mathrm{mm}$. Axial force remains unchanged up to reduce the thickness of processed layer and the beginning of the fourth stage. The fourth stage was characterized by the end of the cutting process and the release of the wedge from CFRP (Fig. 4).

Numerical simulation found that the angle of orientation of fibers affects the average value of cutting force, the amplitude of oscillation, the depth of damage the cut surface. The smallest impact on the direction of the main cutting motion detected at an angle of fiber orientation $\theta=135^{\circ}$, and minimum depth of damage of the machined surface - at an angle of fiber orientation $\theta=45^{\circ}$. 

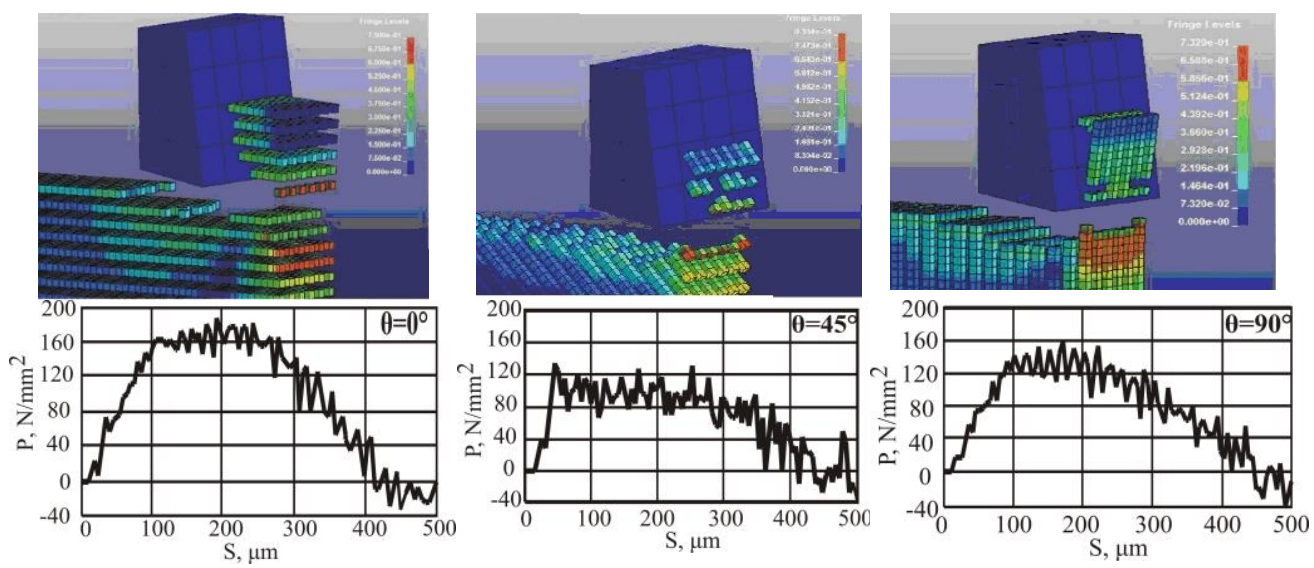

Fig. 4. Influence of fiber orientation on specific cutting force.

The performed calculations showed that the mechanism of chip formation with different fiber orientations is different. Thus, in the model with $\theta=0^{\circ}$, the emergence and development of an advanced crack was revealed, which propagates along the interface between the matrix and the fiber (Fig. 4). The impact of the wedge on the machined material extends before the cutting edge by $200 \mu \mathrm{m}$. The extinction of the crack is evidenced by a decrease in the specific cutting force in the range of $200 \mu \mathrm{m}$ before the output of the tool. Chips formation is due to the detachment of the cut layer from CFRP workpiece. The primary destruction of the material occurs in the matrix. The results show that the least impact of the wedge on the material being processed in the direction of the main motion is observed with a fiber orientation angle $\theta=135^{\circ}$ and is only $100 \mu \mathrm{m}$.

It should be noted that, because of the change in the fracture mechanism, the amplitude of the oscillation of the cutting force in the machining cycle changes with the angle of the fibers. The smallest amplitude is observed at $\theta=0^{\circ}$, the largest at $\theta=135^{\circ}$. Chip formation with an angle $\theta=0^{\circ}$ occurs under the action of compressive forces on the side of the wedge, while cutting with an angle $\theta=135^{\circ}$ bending from the loading cycle. Since the accumulation of damage before destruction in the second case is greater, then the amplitude of the change in force is greater.

Model experiments showed that chip formation is accompanied by periodic destruction of the machined surface. Although the period of damage in all cases was approximately the same (about $180 \mu \mathrm{m}$ ), the depth of damage was different. The greatest depth of damage was fixed at $\theta=0^{\circ}$ and $\theta=90^{\circ}$, i.e. with maximal cutting force. On the other hand, the lowest depth of damage corresponds to the fiber orientation angle $\theta=45^{\circ}$, which provides the lowest cutting force with the smallest amplitude.

\section{Experimental Setup}

Experimental studies of orthogonal cutting were carried out under laboratory conditions at a constant temperature of $20^{\circ} \pm 2^{\circ} \mathrm{C}$. During the experiment, CFRP samples has following geometric parameters width $(b)=20 \mathrm{~mm}$, length $(L)=30 \mathrm{~mm}$ and a height $(h)=7 \mathrm{~mm}$, with fiber orientation angles $\theta=0^{\circ}, \theta=45^{\circ}, \theta=90^{\circ}, \theta=135^{\circ}$. Depth of cut a $=0,1 \mathrm{~mm}$, tool material HSS. Wedge geometry in the orthogonal plane with angles $\gamma=7^{\circ}, \alpha=10^{\circ}$.

During FEM modelling of CFRP orthogonal cutting it was defined that on samples with fiber orientation angle $\theta=0^{\circ}$, fiber pull out was a leading crack was up to $40 \mu \mathrm{m}$ depth and $180 \mu \mathrm{m}$ length accompanied by a decrease in the specific cutting force. During experimental study this damaged of machined surface was detected length of $1.5 \mathrm{~mm}$ and a width of $0.5 \mathrm{~mm}$ (Fig. 5). A detailed examination of the detected defect made it possible to establish that the surface of the depression formed by drawing fibers is not homogeneous and abounds with a large number of irregularities, broken and crumbled fibers. The presence of these defects is explained by the fact that chip formation was accompanied by an interlayer shift, with a low value of plastic deformations, and the parameters of the cutting process during the formation of such chips are determined by the properties of CFRP and its ability to resist shear in the reinforcement plane. Because of machining, element chip with a fragment size of 2 to $5 \mathrm{~mm}$ were obtained. 

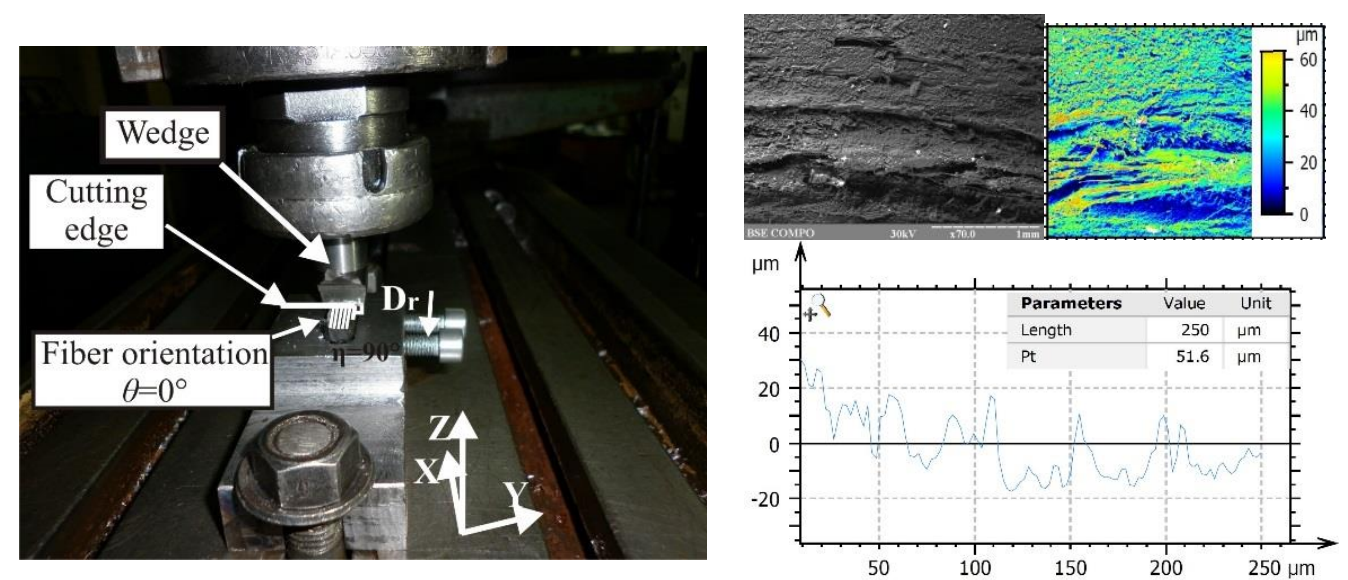

Fig. 5. Experimental results of CFRP samples orthogonal cutting with fiber orientation $\theta=0^{\circ}$.

During FEM modelling of CFRP orthogonal cutting it was defined that on samples with fiber orientation angle $\theta=45^{\circ}$ was characterized fiber pull out up to $10 \mu \mathrm{m}$ with a period of damage of $180 \mu \mathrm{m}$. Analysis of the images of the treated surface confirmed that orthogonal cutting of CFRP samples with an angle $\theta=45^{\circ}$ was not accompanied by significant damage (Fig. 6); nevertheless, the surface formed upon the destruction of the cut layer is smooth with considerable "smearing" of the matrix material due to contact of the tool clearance surface with machined surface, as a consequence of the elastic restoration of the matrix. Because of orthogonal cutting of samples with an angle of fiber orientation $\theta=45^{\circ}$, large cylindrical chips were obtained with a width of $5 \mathrm{~mm}$ and a length of $15 \mathrm{~mm}$. Plastic deformation of the material was realized at interlayer shear with compression.
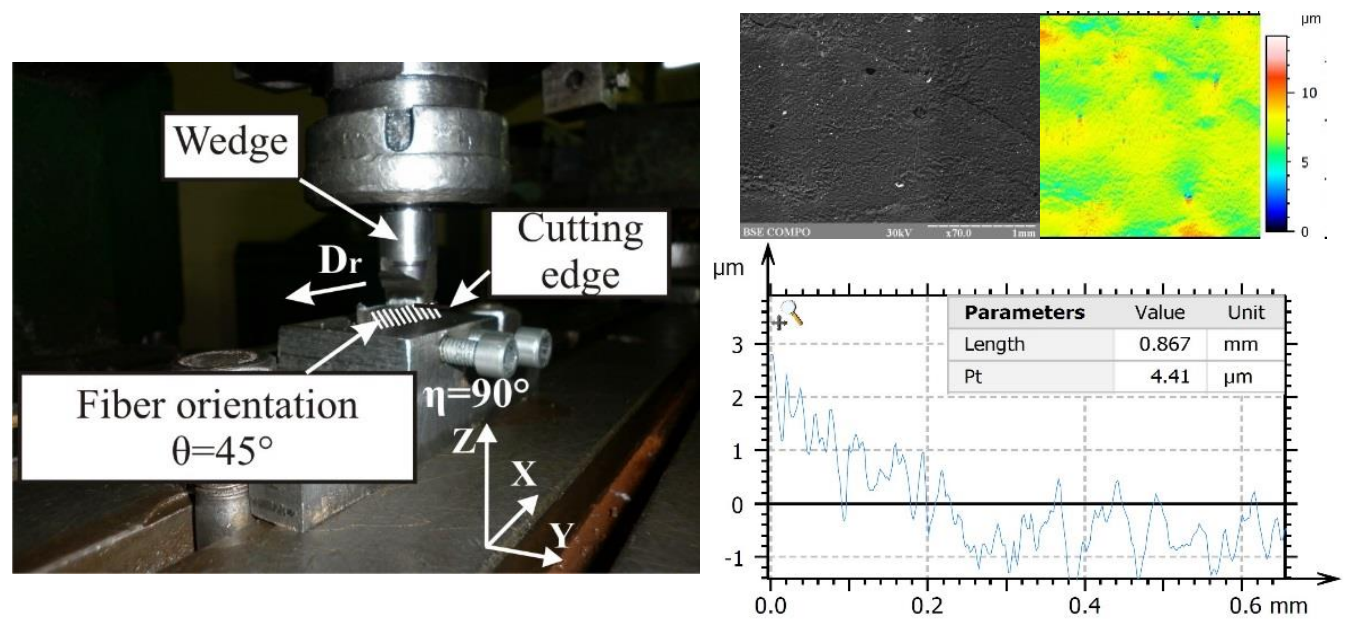

Fig. 6. Experimental results of CFRP samples orthogonal cutting with fiber orientation $\theta=45^{\circ}$

During FEM modelling it was established that samples with fiber orientation angle $\theta=90^{\circ}$ has fiber pull out at machined surface up to $30 \mu \mathrm{m}$. During orthogonal cutting of CFRP samples with the same angle of fiber orientation, based on the analysis of electron microscope photos, it was found that chip of CFRP was formed under longitudinal bending mechanism. Under that circumstance the formation of a shear crack along the interface of the "fibrous filler-matrix" was provoked (Fig. 7).

As the results of the simulation show, the processing of samples with an angle $\theta=135^{\circ}$ is accompanied by pull out of the fibers to $35 \mu \mathrm{m}$, significant oscillations in the cutting force, delamination, shear of fibers, and out-of-plane displacement. The effect of the cutting edge on the side of the fibers causes them to be substantially bent, which triggers the formation of cracks and the formation of long chip elements. Due to the destruction under the cutting surface, the thickness of the chips is much greater than the thickness of the cut. This is confirmed by a fragment of chips obtained during the full-scale experiment, reaching a length of more than 10 $\mathrm{mm}$, and a width of 1 to $3 \mathrm{~mm}$. Inspection of the treated surface showed that it contains areas with elongated and not cut fibers of a length from 0.7 to $1.2 \mathrm{~mm}$ and a width of 0.5 to $0.7 \mathrm{~mm}$. 

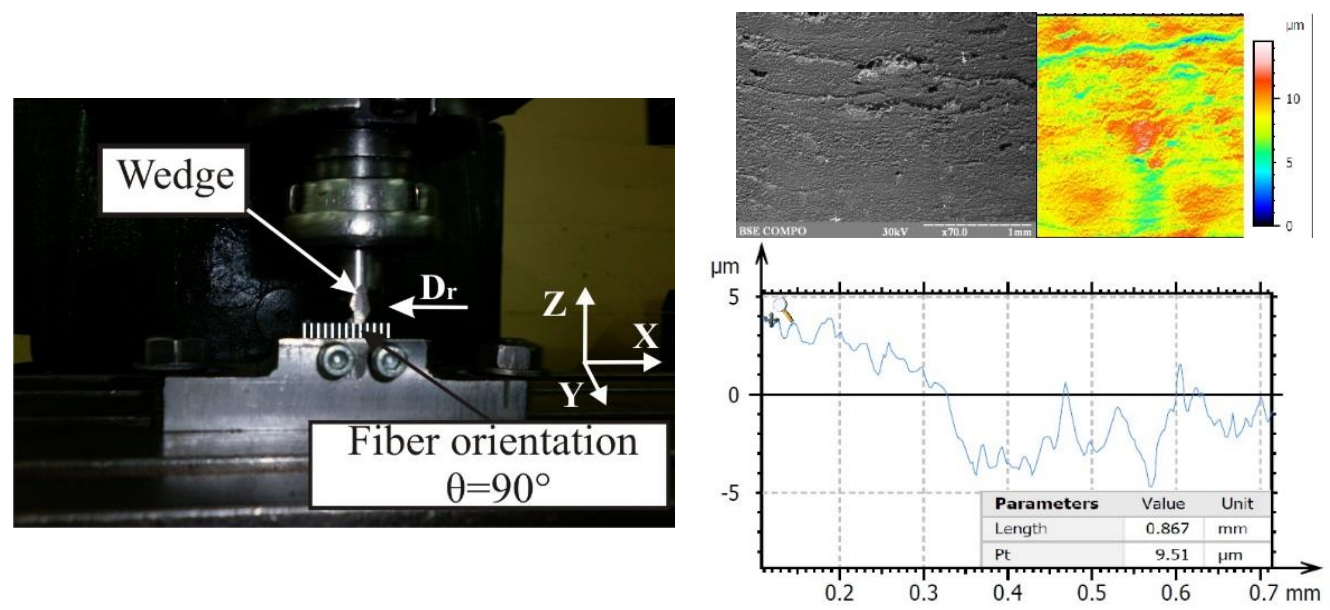

Fig. 7. Experimental results of CFRP samples orthogonal cutting with fiber orientation $\theta=90^{\circ}$

\section{Conclusions}

It was determined that the influence of the fiber orientation on the quality of the machined surface was studied in the orthogonal plane, while the presence of a spatial orientation of the fiber was established, which determines the existence of angles that determine the position of the fiber relative to other planes, which also affects the quality of the machined surface. The scheme and the physical meaning of the angles of the spatial orientation of the fiber are formulated. By the finite element method (FEM) and during the full-scale experiment, the effect of the fiber orientation angle $\theta$ and the orientation angle of the cutting edge was studied. The influence of the fiber orientation angle and the orientation angle of the cutting edge with respect to the direction of reinforcement is investigated. Using the FEM, it was found that the lowest damage of fiber pull pit was observed at $\theta=45^{\circ}$, which was confirmed during experiments of orthogonal cutting of unidirectional CFRP samples. The greatest damage was detected at $\theta=0^{\circ}$, although according to the results of machining the greatest amount of damage in the form of fiber pull out and not cut fibers was found in samples with angles $\theta=135^{\circ}$. Comparing the results of the model and full-scale experiment, it can be concluded that the most qualitative surface without visible damage was obtained after machining sample with angle of fiber orientation $\theta=45^{\circ}$.

Acknowledgments. The authors appreciate the financial support of scholarship stay from Slovak Academic Informational Agency (SAIA) in the frame of National Scholarship Progamme of the Slovak Republic (NSP) and State Fund for Fundamental Research of Ukraine in the frame of Research Grant № 0117U002252.

\section{References}

[1] Arrazola, P. J. et al.: Recent advances in modelling of metal machining processes. CIRP Annals - Manufacturing Technology, Vol. 62, pp. 695-718 (2013)

[2] Murčinková, Z., Vasilko, K.: Thermo-physical aspects of chip machining, High Temperatures High Pressures, Vol. 45 (4), pp. 273-289 (2016)

[3] Vasilko, K., Mádl, J.: Teorie obrábění (Theory of cutting). University of J.E. Purkyně, Ústínad Labem (2012)

[4] Wang, C., et al.: Occurrence and formation mechanism of surface cavity defects during orthogonal milling of CFRP laminates. Composites Part B: Engineering, Vol. 109, pp. 10-22 (2017) [5] Feito, N., et al.: Numerical prediction of delamination in CFRP drilling. Composite Structures, Vol. 108, pp. 677-683 (2014) 